\title{
KARIM MESSEGHEM
}

SYLVIE SAMMUT

Univ. de Montpellier, MRM, Labex Entreprendre, Institut Montpellier Management

\section{LEILAA TEMRI}

UMR MOISA, Montpellier SupAgro

\section{ÉTIENNE ST-JEAN}

INRPME UQTR (Québec, Canada)

\section{Les mutations de l'accompagnement entrepreneurial}

$\mathrm{L}$

9accompagnement entrepreneurial connaît depuis une dizaine d'années des transformations majeures. Portée par une forte dynamique entrepreneuriale et la diffusion d'une culture start-up, l'industrie de l'accompagnement entrepreneurial subit de profondes mutations (Bakkali et al., 2013 ; Calmé et al., 2016 ; Commission nationale d'évaluation des politiques d'innovation, 2016). De nouveaux dispositifs (accélérateurs, espaces de coworking, pré-incubateurs étudiants, incubateurs sociaux, etc.) fleurissent ici ou là. De nouveaux entrants issus de la sphère privée et souvent empreints d'une culture nordaméricaine (Station F, The Family, Founder Institute, etc.) dynamisent le secteur et le contraignent à une remise en question. En parallèle, des modèles d'entrepreneuriat novateurs tels que l'entrepreneuriat hybride ou la gig economy se développent. 
L'entrepreneuriat n'est plus forcément pensé en termes de carrière individuelle, de long terme. Les profils d'entrepreneurs se singularisent, se diversifient. L'entrepreneuriat féminin se développe. Le repreneuriat connaît un fort engouement (Boumedjaoud et al., 2019). En outre, de nouveaux outils de création de connaissances tels que les Fab Labs ou les Living Labs démocratisent l'accès aux connaissances qui sont sources d'innovation. Par ailleurs, l'accès aux ressources financières est facilité, notamment depuis l'apparition du crowdfunding (Drover et al., 2017), sans compter l'accroissement des projets suivant une logique de lean start-up, réduisant du coup les besoins au démarrage. Toutes ces évolutions invitent à repenser l'accompagnement entrepreneurial. Si la littérature académique se fait l'écho de certaines de ces transformations (Vanderstraeten et Matthyssens, 2012 ; Messeghem et al., 2013 ; Baraldi et Havenvid, 2016), ces mutations suscitent néanmoins de nombreuses questions, à différents niveaux. Comment ces nouveaux dispositifs, ces nouveaux entrants, ces nouvelles formes d'entrepreneuriat, cet accès démultiplié aux connaissances, modifientelles la forme, le contenu et la mission de l'accompagnement entrepreneurial ? Dans un contexte de diminution des ressources publiques (Economic Commission for Europe 2001 ; France Stratégie, 2016 ; Messeghem et al., 2018), dans quelle mesure les politiques publiques d'accompagnement entrepreneurial se révèlent-elles efficientes, efficaces et effectives ? Dans une perspective macro, méso et micro-économique, dans quelles mesures les écosystèmes de l'accompagnement entrepreneurial contribuent-ils à la dynamique d'entrepreneuriat et d'innovation (Ben Letaifa et Rabeau, 2012 ; Mina et al., 2015 ; Roundy et al., 2017 ; Theodoraki et Messeghem, 2017 ; Theodoraki et al., 2018). L'ensemble de ces questions interroge de multiples théories comme l'approche fondée sur les ressources (RBV), la théorie des capacités dynamiques, la théorie de l'alignement stratégique, la théorie du capital social, etc.

\section{POUR UNE LECTURE MULTINIVEAU DES MUTATIONS DE L'ACCOMPAGNEMENT ENTREPRENEURIAL}

Les mutations de l'accompagnement entrepreneurial peuvent être abordées en retenant une lecture multiniveau (Baraldi et Havenvid, 2016 ; Theodoraki et Messeghem, 2017).

$\mathrm{Au}$ plan macro-économique, la question peut être traitée sous l'angle des réseaux d'accompagnement nationaux et internationaux qui jouent un rôle déterminant en favorisant le développement d'actions et de stratégies collectives. Ces réseaux nationaux et internationaux sont propices à l'échange de bonnes pratiques. Quel est l'impact effectif de ces réseaux sur les pratiques d'accompagnement ou sur les modes de gestion des structures ? Plus globalement, on doit aussi s'interroger sur l'impact des dispositifs de soutien et d'accompagnement sur le développement économique des territoires, dans la mesure ou leur existence même est parfois remise en cause par les acteurs politiques et économiques, voire les chercheurs (Shane, 2008). $\mathrm{Au}$ plan méso, il est nécessaire de se concentrer également sur la coordination des dispositifs et l'interaction des écosystèmes intermédiaires (Simatupang et al., 2015). Plus globalement, comment s'articulent les niveaux locaux, nationaux et 
internationaux, dans la mesure où de nombreux dispositifs s'entremêlent à différents niveaux ? Cette perspective peut également être abordée sous l'angle de l'industrie de l'accompagnement. Soumise à la pression des nouveaux entrants et à un phénomène de convergence qui amplifie cette émergence de nouveaux acteurs (Fabbri et Charue-Duboc, 2013 ; St-Jean et El Agy, 2013 ; Mian et al., 2016 ; Pauwels et al., 2016 ; Garrett et al., 2017), comment les nouveaux dispositifs et les nouveaux entrants vont-ils modifier les écosystèmes entrepreneuriaux ? Comment vont-ils s'articuler entre eux : Concurrence ? Coopération? Coopétition ? (Theodoraki et Messeghem, 2015). Quels modes de coordination vont-ils mettre en œuvre ?

De façon concomitante, le digital conduit à redéfinir les frontières de cet écosystème de l'accompagnement entrepreneurial (Mercuri-Chapuis et Gauthier, 2016). Quelles sont les nouvelles pratiques d'accompagnement qu'il engendre ? Quel impact sur l'industrie de l'accompagnement ? Cette industrie n'est-elle pas à l'aube d'un phénomène d'ubérisation comme le suggèrent certains acteurs de l'accompagnement (Labex Entreprendre Publication, 2017) ? Ce phénomène va-t-il amener les acteurs traditionnels à modifier leurs pratiques d'accompagnement ?

Enfin, au plan micro, si la plupart des pays européens ont privilégié pendant longtemps un modèle fondé sur des financements publics, cette approche tend à être remise en question (Bakkali et al., 2013 ; Pauwels et al., 2016 ; Baraldi et Havenvid, 2016 ; Maus et Sammut, 2017a). Les acteurs historiques doivent repenser leur modèle d'affaires (Maus et Sammut, 2017b ; Maus, 2019) en s'inspirant de ceux des nouveaux entrants mais aussi en retravaillant leur mission originelle. Avec comme trame de fond la multiplication des formations universitaires en entrepreneuriat ou l'accès à de nombreuses informations disponibles en ligne, les besoins des nouvelles générations fraichement formées pourraient être bien différents et exiger un passage d'un accompagnement de type « transmission de contenu » à celui, plutôt, de soutien au processus de création, voire de « soutien à la problématisation » (Schmitt, 2015). Parallèlement, le développement de l'entrepreneuriat social génère de nouveaux comportements, tels l'essaimage de structures d'accompagnement, encore peu explorés.

Dans ce contexte et compte tenu des nouveaux modes d'accès aux connaissances et aux nouvelles formes d'entrepreneuriat, comment évoluent les besoins des nouveaux entrepreneurs ? Quelles seront les nouvelles pratiques d'accompagnement mises en place pour y répondre ? Quelles seront les nouvelles compétences (Bakkali et al., 2010) et les nouveaux outils à développer ? Les porteurs de projets se retrouvent face à une variété d'acteurs ayant des missions diverses, dont certaines se recoupent sans forcément se compléter parfaitement. Que comprennent-ils de cette offre et de ces acteurs quant à leur capacité à répondre à leurs besoins ? Face aux différents acteurs d'un territoire, comment se gère la coordination entre les besoins de la personne et de son projet ? Quel est l'impact de cette coordination sur le processus entrepreneurial ? Au final, ces mutations favorisentelles des écosystèmes d'accompagnement plus durables ?

Ce dossier spécial n'a pas pour ambition de répondre à toutes ces questions. L'étendue est bien trop large mais il pose les 
fondements d'une réflexion de long terme sur le devenir et la cohabitation d'écosystèmes et d'acteurs aux profils et missions devenues hétérogènes.

\section{LES CONTRIBUTIONS DE CE DOSSIER}

Dans les quatre contributions de ce dossier, deux se rapportent à des situations particulières d'accompagnement, des femmes pour le premier article, des repreneurs pour le second. Le troisième traite de l'écosystème entrepreneurial, et enfin le quatrième se rapporte aux modalités de financement.

Marie-Hélène Duchemin et Séverine Lemarié-Quillerier abordent la question de l'accompagnement de l'entrepreneuriat au féminin par une entrée originale : la contribution de la mémoire autobiographique. Les auteures constatent en effet une croissance des recherches consacrées à l'accompagnement des femmes entrepreneurs, car leur décision apparaît davantage contrainte que chez les hommes, et, en particulier, liée à leur expérience professionnelle antérieure. L'objectif de l'article est ainsi de comprendre comment les mécanismes de souvenir du salariat interviennent dans le choix de création d'entreprise des entrepreneures, et déterminer quelles conséquences peuvent en être tirées en matière d'accompagnement à la création d'entreprise de ces femmes. La mémoire autobiographique est un concept issu des neurosciences, qui permet d'étudier le souvenir, c'est-à-dire, ici, la reconstruction d'événements passés. Les auteures montrent que dans le cadre d'un accompagnement collectif avec d'autres créatrices d'entreprises, le recours à la mémoire de leur expérience salariée antérieure, conjointement avec les autres créatrices, les aide à se projeter dans leur future activité et à lever un certain nombre d'obstacles. La recherche, exploratoire, est qualitative, et se base sur une série d'entretiens ouverts et auto-administrés auprès de 116 entrepreneures. Les résultats montrent en premier lieu que si le salariat a permis aux femmes entrepreneures d'acquérir une solide expérience, plusieurs entraves les ont incitées à se lancer dans l'entrepreneuriat, qui devient ainsi contraint. En second lieu, il apparaît que pour la plupart d'entre elles, c'est un souvenir spécifique qui a réellement orienté leur choix de l'entrepreneuriat. Enfin, l'analyse des similitudes par l'intermédiaire de l'indice de Cramer sur des données primaires recueillies dans un incubateur, montre que la mémoire autobiographique, par le souvenir saillant raconté à d'autres créatrices dans la même situation, vient conforter le rôle de l'accompagnement collectif.

L'article de Bérangère Deschamps et Thierry Lamarque traite quant à lui de l'accompagnement du candidat repreneur d'entreprise dans la phase de négociation, en focalisant sur les reprises externes. Cette phase fait l'objet de peu d'attention dans la littérature, malgré son caractère crucial dans la réussite du processus de transfert. Certes, les « suspects » traditionnels sont connus pour y intervenir, tels les avocats, notaires et comptables. Mais considérant que ce processus implique des moments critiques et des émotions diverses, pouvant à tout moment faire dérailler celui-ci, le recours à d'autres spécialistes pourrait être judicieux dans ce contexte, que ce soient des mentors, coachs ou conseillers spécialisés, notamment. Pour obtenir une vision plus étoffée sur ce qui s'y déroule, les auteurs ont étudié 35 négociations de transferts d'entreprises en observation participante et mobilisé les 
entretiens semi-directifs de dix repreneurs impliqués dans des négociations de transferts externes. En abordant l'analyse de leurs documents sous l'angle des différentes phases de la négociation, ils constatent d'entrée de jeu des émotions nombreuses et puissantes, culminant bien souvent dans un climat de tension évident. Il s'avère alors important pour le repreneur de considérer l'apport d'un conseiller pertinent sur la dimension humaine. À cet égard, les auteurs montrent le rôle du « conseil acheteur », qui complète les compétences accessibles au repreneur et lui permet, à toutes les étapes $\mathrm{du}$ processus, de dénouer ou même « déjouer » certaines impasses, de par son expérience de situations similaires. Il fait partie de la « coalition repreneur », autant active sur le devant scène de la table de négociation que dans les réunions internes où s'élabore la stratégie de reprise. Il se situe alors à l'intersection des aspects humains et techniques de la négociation, entre les conseils généralistes et ceux d'experts, dépassant alors les aspects «techniques » traditionnellement associés à la négociation. Cet article constitue un apport important à notre compréhension des rôles des différents acteurs de l'accompagnement impliqués dans les transferts d'entreprises.

L'article de Éric Persais aborde la question du développement des écosystèmes entrepreneuriaux (EE) en se focalisant sur le rôle des établissements d'enseignement supérieur et de recherche (ESR), un aspect négligé des travaux sur la question. En particulier, il $\mathrm{y}$ est question de leur apport en termes de compétences, notamment dans le fait de susciter l'intérêt des étudiant.e.s envers l'entrepreneuriat, mais également dans le fait de procurer les capitaux humains requis aux projets entrepreneuriaux qui s'y développent. L'auteur se pose la question $\mathrm{du}$ caractère inclusif des écosystèmes entrepreneuriaux pour expliquer leurs réussites et focalise son analyse sur un cas unique d'incubateur de la ville de Niort (France) dans le domaine de l'AssurTech (virage numérique des compagnies d'assurance) : Niort Tech.

$\mathrm{Au}$ travers de ce cas, on peut identifier plusieurs enjeux de développement d'un écosystème qui est principalement orienté pour soutenir une industrie oligopolistique, et dont les acteurs et actrices économiques favorisent les éléments de territorialité, notamment de par la concentration géographique des acteurs concernés. Selon les acteurs concernés, il semble largement préférable que cet $\mathrm{EE}$ se développe hors des institutions d'ESR, tout en conservant un caractère inclusif pour favoriser l'adhésion des différents acteurs. Dans un contexte où les établissements d'ESR évoluent dans des logiques différentes de celles des autres entités impliquées, l'entrepreneuriat constituerait néanmoins un vecteur de convergence d'intérêts pour favoriser l'inclusion des établissements d'ESR. L'auteur souligne aussi la participation active des entreprises aux conseils de perfectionnement des formations ainsi que l'intérêt affiché par certains de tendre vers une recherche qui se veut appliquée, deux aspects qui semblent importants à cet égard. Si aujourd'hui les établissements d'ESR jouent un rôle de support à l'écosystème entrepreneurial qui se développe dans cette région, ceux-ci n'en demeurent pas moins centraux pour la suite, d'où l'importance pour les EE qui se développent de conserver un caractère inclusif, impactant alors les dynamiques d'accompagnement en leur sein. 
Enfin, l'entrepreneuriat bénéficie de nouveaux modes de financement. Parmi ceuxci, le crowfunding se développe depuis le début des années 2010. Il s'agit d'un mode de financement participatif, auquel un nombre croissant d'entrepreneurs recourt. Pourtant, si certains travaux montrent que l'activité des contributeurs, au sein de ces plateformes, peut donner lieu à la construction d'un réseau social qui dépasse les projets individuels, il n'y a pas d'étude disponible concernant les caractéristiques de ces réseaux, ni leur impact sur le taux de succès des campagnes de levées de fonds. C'est à ces deux aspects, fortement liés, qu'est consacré l'article de Ima Lyubareva, Laurent Brisson, Cécile Bothorel et Romain Billot. À travers le cas de la plateforme de don Ulule, l'un des premiers sites européens de crowdfunding, il s'agit d'identifier le réseau social inter-projets qui se constitue autour de la plateforme, et de montrer le rôle de celui-ci dans le succès de campagnes de levées de fond. A cet effet, l'analyse des réseaux sociaux (ARS) est mobilisée. Elle permet d'identifier les interactions sociales non observables directement, et donc le réseau social de la plateforme, au-delà des projets individuels. Elle est combinée avec d'autres méthodes, plus classiques, de l'analyse de données et de l'économétrie. Les données des cinq premières années de fonctionnement d'Ulule sont exploitées, ce qui, après filtrage des données, représente 19544 projets, rassemblant 876758 contributeurs. 11900 projets ont été financés avec succès. Cette méthodologie originale permet de valider les hypothèses énoncées dans l'article à savoir : les projets financés par des membres appartenant au réseau social de Ulule ont un taux de succès significativement supérieur par rapport à ceux n'y appartenant pas. De plus, un effet thématique est constaté : la présence des groupes est plus fréquente et impacte particulièrement les projets thématiques ayant une forte composante communautaire dans la production et dans la consommation des biens. En second lieu, cinq groupes de contributeurs ont pu être identifiés à partir d'un certain nombre de caractéristiques. Il apparaît que ceux ayant une activité sociale plus importante contribuent davantage en termes de nombre de projets ou de montants investis. Ils impactent également le taux de succès des campagnes.

Au total, ces contributions traitent quelquesunes des nouvelles problématiques de l'accompagnement entrepreneurial, à différents niveaux, en mobilisant des méthodologies originales. Une part importante est accordée à la psychologie des créateurs d'entreprises, à leurs émotions, mais aussi aux liens entre entrepreneurs et entre financeurs sur les plateformes, par exemple. L'entrepreneuriat est ainsi largement abordé sous un angle humain dans ces contributions, aspects psychologiques, capital humain, réseaux sociaux, mais aussi compétences. 


\section{BIBLIOGRAPHIE}

Bakkali C., Messeghem K. et Sammut S. (2010). « Les structures d'accompagnement à la création d'entreprise à l'heure de la gestion des compétences », Management et Avenir, $n^{\text {o } 39, ~ p . ~ 149-162 . ~}$

Bakkali C., Messeghem K. et Sammut S. (2013). « Pour un outil de mesure et de pilotage de la performance des incubateurs », Management international, vol. 17, $\mathrm{n}^{\mathrm{o}}$ 3, p. 140-153.

Baraldi E. et Havenvid M.I. (2016). "Identifying new dimensions of business incubation: A multi-Level analysis of Karolinska Institute's incubation system”, Technovation, vol. 50-51, p. 53-68.

Ben Letaifa S. et Rabeau Y. (2012). « Evolution des relations coopétitives et rationalités des acteurs dans les écosystèmes d'innovation », Management International, vol. 16, $\mathrm{n}^{\circ} 2$, p. 57 84.

Boumedjaoud D., Cloutier L. et Messeghem K. (2019). Regards des repreneurs sur La reprise d'entreprise : Le cas des lauréats de Réseau Entreprendre, Rapport Labex Entreprendre, Montpellier.

Calmé I., Onnée S. et Zoukoua É. (2016). « Plateformes de crowdfunding et acteurs de l'écosystème entrepreneurial », Revue française de gestion, vol. 42, n 259, p. 139-154.

Commission nationale d'évaluation des politiques d'innovation (2016). Quinze ans de politiques d'innovation en France, Paris.

Drover W., Busenitz L., Matusik S., Townsend D., Anglin A. et Dushnitsky G. (2017). "A review and road map of entrepreneurial equity financing research: Venture capital, corporate venture capital, angel investment, crowdfunding, and accelerators", Journal of Management, vol. 43, $\mathrm{n}^{\mathrm{o}}$ 6, p. 1820-1853.

Economic commision for Europe. (2001). Best practice in business incubation, New York and Geneva.

Fabbri J. et Charue-Duboc F. (2013). « Un modèle d'accompagnement entrepreneurial fondé sur des apprentissages au sein d'un collectif d'entrepreneurs : le cas de La Ruche », Management international, vol. 17, $\mathrm{n}^{\mathrm{o}} 3$, p. 87-99.

France Stratégie. (2016). «Quinze ans de politiques d'innovation en France », Commission nationale d'évaluation des politiques d'innovation, janvier.

Garrett L.E., Spreitzer G.M. et Bacevice P.A. (2017). "Co-constructing a sense of community at work: the emergence of community in coworking spaces", Organization Studies, vol. 38, $\mathrm{n}^{\mathrm{o}} 6$, p. 821-842.

Hackett S.M. et Dilts D.M. (2004b). "A real options-driven theory of business incubation”, Journal of Technology Transfer, vol. 29, $\mathrm{n}^{\circ}$ 1, p. 41-54.

Labex Entreprendre Publications (2017). « Ubérisation : quels défis pour l'accompagnement entrepreneurial ? », $\mathrm{n}^{\mathrm{o}} 19$, www.labex-entreprendre.fr 
Maus A. et Sammut S. (2017a). « Les structures d'accompagnement, des entreprises comme les autres. Contribution de la théorie des capacités », Gestion 2000, vol. 34, n 5, p. 203-226.

Maus A. et Sammut S. (2017b). "Evolution in incubators' Business Model, the contribution of individual and collective sensing of opportunities and dynamic capabilities", Frontiers of Entrepreneurship Research, Babson Publication, vol. 37, p. 202-207.

Maus A. (2019) Evolution des business models d'incubateurs. Contribution de la théorie des capacités dynamiques, Thèse de doctorat en Sciences de Gestion, Université de Montpellier.

Mercuri-Chapuis S. et Gauthier T. (2016). « L'économie collaborative en Suisse romande à l'horizon 2030 : les MOOCs à l'heure de Coursera », Gestion 2000, vol. 34, nº 5, p. 55-73.

Messeghem K., Carrier C., Sammut S., Thurik R. et Chabaud D. (2013). « L'accompagnement entrepreneurial, une industrie en quête de leviers de performance ? », Management international, vol. 17, $\mathrm{n}^{\mathrm{o}} 3$, p. 65-71.

Messeghem K. et Sammut S. (2017). "Editorial: Thirty years of research in entrepreneurial support", International Journal of Entrepreneurship and Small Business Journal, vol. 23, $\mathrm{n}^{\mathrm{o}} 4$, p. 405-418.

Messeghem K., Bakkali C., Sammut S. et Swalhi A. (2018). "Measuring nonprofit incubator performance: Toward an adapted balanced scorecard approach", Journal of Small Business Management, vol. 56, $\mathrm{n}^{\mathrm{O}}$ 4, p. 658-680.

Mian S., Lamine W. et Fayolle A. (2016). “Technology business incubation: An overview of the state of knowledge", Technovation, vol. 50-51, p. 1-12.

Mina A., Dagnino G.B. et Ben Letaifa S. (2015). "Coopetition and cooperation in entrepreneurial ecosystmes: A life-cycle analysis of a Canadian ICT ecosystem”, Innovation, alliances, and networks in high-tech environments, Belussi, F., Orsli L. (Eds), Routledge studies in global competition, Edgar, Cheltenham, UK, p. 65-81.

Pauwels C., Clarysse B., Wright M. et Van Hove J. (2016). "Understanding a new generation of incubation model: The accelerator", Technovation, vol. 50-51, p. 13-24.

Roundy P.T., Brockman B.K. et Bradshaw M. (2017). "The resilience of entrepreneurial ecosystems", Journal of Business Venturing Insights, vol. 8, p. 99-104.

Schmitt C. (2015). « Du réparateur au facilitateur : changement de regard sur l'accompagnement », Entreprendre \& Innover, $\mathrm{n}^{\mathrm{o}} 4$, p. 93-101.

Shane S.A. (2008). The Illusions of Entrepreneurship: The Costly Myths that Entrepreneurs, Investors, and Policy Makers Live by, Yale University Press, New Haven, C.T.

Simatupang T.M., Schwab A. et Lantu D.C. (2015). "Introduction: building sustainable entrepreneurship ecosystems", International Journal of Entrepreneurship and Small Business, vol. 26, $\mathrm{n}^{\mathrm{o}} 4$, p. 389-398.

St-Jean E. et El Agy M. (2013). « La motivation à être bénévole des mentors influence-t-elle la relation de mentorat ? Une étude en dyade dans un contexte d'entrepreneurs novice », Management International, vol. 17, n $\mathrm{n}^{\mathrm{O}}$ 3, p. 112-125. 
Theodoraki C. et Messeghem K. (2015). «Écosystème de l'accompagnement entrepreneurial : une approche en termes de coopétition », Entreprendre \& Innover, $\mathrm{n}^{\circ} 23$, p. 102-111.

Theodoraki C. et Messeghem K. (2017). "Exploring the entrepreneurial ecosystem in the field of entrepreneurial support: A multi-level approach", International Journal of Entrepreneurship and Small Business, vol. 31, $\mathrm{n}^{\mathrm{o}}$ 1, p. 47-66.

Theodoraki C., Messeghem K. et Rice M.P. (2018). "A social capital approach to the development of sustainable entrepreneurial ecosystems: An explorative study", Small Business Economics, vol. 51, n ${ }^{\mathrm{o}}$ 1, p. 153-170.

Vanderstraeten J. et Matthyssens P. (2012). "Service-based differentiation strategies for business incubators: Exploring external and internal alignment", Technovation, vol. 32, $\mathrm{n}^{\mathrm{o}} 12$, p. 656-670. 